\title{
Characterization of the eLine ASICs in prototype detector systems for LCLS
}

\author{
G. A Carini*, A. Dragone, B.-L. Berube, P. Caragiulo, D. M. Fritz, P. A. Hart, R. Herbst, S. Herrmann, C. J. Kenney, \\ A. J. Kuczewski, H. T. Lemke, J. Mead, J. Morse, J. Pines, A. Robert, D. P. Siddons, D. Zhu and G. Haller
}

\begin{abstract}
Line", a class of multichannel time-variant integrating front-end Application Specific Integrated Circuits (ASICs), has been completed at SLAC National Accelerator Laboratory for applications at the Linac Coherent Light Source (LCLS). The class, designed for pixelated sensors with columnparallel readout, is composed of two front-end ASICs: one designed for high-dynamic range applications (eLine10k) and one designed for ultra-low noise applications (eLine100). The first allows large input full-scale signals, on the order of $10^{4} 8 \mathrm{keV}$ photons, with a resolution of half a photon FWHM; while the second provides low noise charge integration, up to a full-scale signal of $1008 \mathrm{keV}$ photons, with an equivalent noise charge (ENC) of 55e- r.m.s. Three different prototype systems utilizing the ASICs are described. The first is a 32k-pixel X-ray Active Matrix Pixel Sensor (XAMPS) detector developed at Brookhaven National Laboratory (BNL) for the X-ray Pump Probe instrument (XPP) at LCLS. The XAMPS are monolithic detectors with fast-frame readout and large full-scale signal. In particular, they provide a full well capacity on the order of $10^{4}$ $8 \mathrm{keV}$ photons per pixel and a resolution of half a photon FWHM. The second prototype, developed around eLine10k, is a beam finder with high dynamic range. The third prototype is developed around eLine100 to be used as detector in a spectrometer. Applications, test results and performance are discussed.
\end{abstract}

\section{INTRODUCTION}

new "eLine" class of multichannel time-variant A integrating front-end Application Specific Integrated Circuits (ASICs) has been completed at SLAC National Accelerator Laboratory for applications at the Linac Coherent Light Source (LCLS). The unique characteristics of fourth generation light sources, namely their pulsed nature and extremely high peak brilliance, demand a dedicated detector development effort. Some common features, such as the fast readout, are intrinsically dictated by the source structure. Noise requirements and full-scale signal capability vary with the experiment. The two ASICs in the eLine class, eLine10k $[1,2]$ and eLine100 [3], cover applications with very different requirements as demanded by LCLS experiments. The first allows large input full-scale signals, on the order of $10^{4} 8 \mathrm{keV}$ photons, with a resolution of half a photon FWHM; the second provides low noise charge integration, up to a full-scale signal

Manuscript received November 16, 2012. SLAC-PUB-15276.

G. A. Carini", A. Dragone, P. Caragiulo, D. M. Fritz, P. A. Hart, R. Herbst, S. Herrmann, C. J. Kenney, H. T. Lemke, J. Pines, A. Robert, D. Zhu and G. Haller are with SLAC National Accelerator Laboratory, Menlo Park, CA ("corresponding author, telephone: 650-926-5009, e-mail: carini@slac.stanford.edu). B.-L. Berube is with the Universite' de Sherbrooke, Sherbrooke, Quebec, Canada. A. J. Kuczewski, J. Mead and D. P. Siddons are with Brookhaven National Laboratory, Upton, NY. J. Morse is with the European Synchrotron Radiation Facility, Grenoble, France. of $1008 \mathrm{keV}$ photons, with an Equivalent Noise Charge (ENC) of $55 \mathrm{e}^{-}$r.m.s. The ASICs are fabricated in TSMC CMOS $0.25 \mu \mathrm{m}$ technology with 64 and 96 channels respectively. A charge pump scheme implementing a zero-balance measurement method [1 - 3] used in both ASICs is their characteristic feature. It provides on-chip coarse analog to digital conversion of the signals and a measurement of the residues with the required resolution. The residues are converted with external 14-bit ADCs.

eLine is the first example of a class of ASICs for LCLS sharing a common back-end section and interface to the rest of the system. During the first years of operation at LCLS, the need for several new large area detectors arranged in different form factors and shapes, and supporting a wide spectrum of experiments has been apparent. Modular, scalable designs are a must. To simplify integration and reduce development time, detectors have been designed around common platforms so that dedicated sensing heads could be incorporated in a common detector system. Detectors based on this platform are then firmware compatible and easy to integrate in the LCLS DAQ system.

TABLE I

ELINE10K PERFORMANCE

\begin{tabular}{ll}
\hline \hline Technology & $\mathrm{TSMC} 0.25 \mu \mathrm{m}$ \\
Die Area & $6 \mathrm{~mm} \times 3.5 \mathrm{~mm}$ \\
Number of Channels & 64 \\
Optimum Input Load & $15 \mathrm{pF}$ \\
Programmable Gain & $5 \mathrm{mV} / \mathrm{fC}$ or $2.5 \mathrm{mV} / \mathrm{fC}$ \\
ENC & $480 \mathrm{e}^{-}$r.m.s. @ $15 \mathrm{pF}$ \\
Maximum Signal & $26 \mathrm{Me}^{-}(12 \mathrm{k}$ photons @ $8 \mathrm{keV})$ \\
Dynamic Range & $95 \mathrm{~dB}$ \\
Power Consumption & $3 \mathrm{~mW} / \mathrm{channel}$ \\
Speed & $500 \mathrm{k}$ pixel/s/channel \\
\hline
\end{tabular}

TABLE II

ELINE100 PERFORMANCE

\begin{tabular}{ll}
\hline \hline Technology & $\mathrm{TSMC} 0.25 \mu \mathrm{m}$ \\
Die Area & $6.8 \mathrm{~mm} \times 5 \mathrm{~mm}$ \\
Number of Channels & 96 \\
Optimum Input Load & $3 \mathrm{pF}$ \\
Gain & $58 \mathrm{mV} / \mathrm{fC}$ \\
ENC & $55 \mathrm{e}^{-}+8 \mathrm{e}^{-} / \mathrm{pF}$ r.m.s \\
Maximum Signal & $260 \mathrm{ke}^{-}(120$ photons @ $8 \mathrm{keV})$ \\
Dynamic Range & $74 \mathrm{~dB}$ \\
Power Consumption & $3.5 \mathrm{~mW} /$ channel \\
Speed & $250 \mathrm{k}$ pixel/s/channel \\
\hline
\end{tabular}

In this paper, the performance of the ASICs, summarized in Table I and Table II, are experimentally demonstrated. Three different prototypes have been developed and characterized. 
Results are reported and discussed in the following paragraphs.

\section{XAMPS DETECTOR TESTS AT SSRL AND LCLS}

The eLine10k ASICs (also referred to as FEXAMPS) was originally conceived as readout electronics for X-ray Active Matrix Pixel Sensors (XAMPS) [4, 5]. The XAMPS, developed at Brookhaven National Laboratory (BNL), are monolithic detectors with fast readout and large full-scale signal. In particular, they provide a full well capacity on the order of $10^{4}$ photons of $8 \mathrm{keV}$ per pixel and a resolution of half a photon FWHM. Each pixel of the matrix is $90 \times 90 \mu \mathrm{m}^{2}$ and contains a JFET switch to control charge integration and readout, which is performed in parallel row by row through the eLine10k ASICs. The rows are addressed by another switcher ASIC, which effectively implements the operation of a rolling shutter.

The devices are fabricated in high resistivity n-type silicon substrates with a thickness greater than $400 \mu \mathrm{m}$, and fully depleted through a junction on the photon entrance window. The charges generated by an impinging photon drift to the exit side of the device where they are collected by an implant, constituting the floating electrode of the pixel or the source of the JFET. This region together with the isolated metal layer on its top forms the capacitor in which the charge is stored. To allow $3.5 \mathrm{pC}$ of full well capacity, this region occupies most of the pixel area.

This version of XAMPS was designed for the X-ray Pump Probe (XPP) instrument at LCLS, for which a 1M-pixel camera is currently being produced. Given the $120 \mathrm{~Hz}$ LCLS repetition rate, a frame has to be read out in $\sim 8 \mathrm{~ms}$, which, due to the XAMPS parallel row readout, turns into a readout time of $\sim 8 \mu$ s per row.

$4 \mathrm{k}$ - and 32k-pixel prototypes were fabricated and tested at BNL, satisfying the requirements of large full-scale signal, noise and fast readout $[5,6]$. Here we report the results of measurements performed at the Stanford Synchrotron Radiation Lightsource (SSRL), BL2-2, and at the LCLS XPP instrument with the 32k-pixel prototype camera.

The prototype camera comprises two 128 x 128 sensors tiled and wire-bonded in an aluminum frame. In the ASICsensor bonding scheme, five chips were used to read out the 256 columns: this was required to compensate for the space between the sensors, and resulted in 32 non-connected channels at the end of each sensor. In addition to the analog board on which sensors and front-end electronics are mounted, two other boards complete the system: a digital board with a Virtex5 Field Programmable Gate Array (FPGA) and a high voltage daughter board. The FPGA controls the settings of front-end and switcher ASICs in the analog board, and communicates data at $2.5 \mathrm{Gbs}$ through a SLAC protocol (PGP) implemented in a PCIe-compliant board. A liquid-cooled copper plate is mounted between analog and digital boards to keep the temperature at $\sim 20^{\circ} \mathrm{C}$. The overall system, which can accommodate the full $1 \mathrm{M}$-pixel sensor area, is very compact and can be mounted and precisely aligned in a robotic arm at the XPP instrument (Fig. 1).

a)

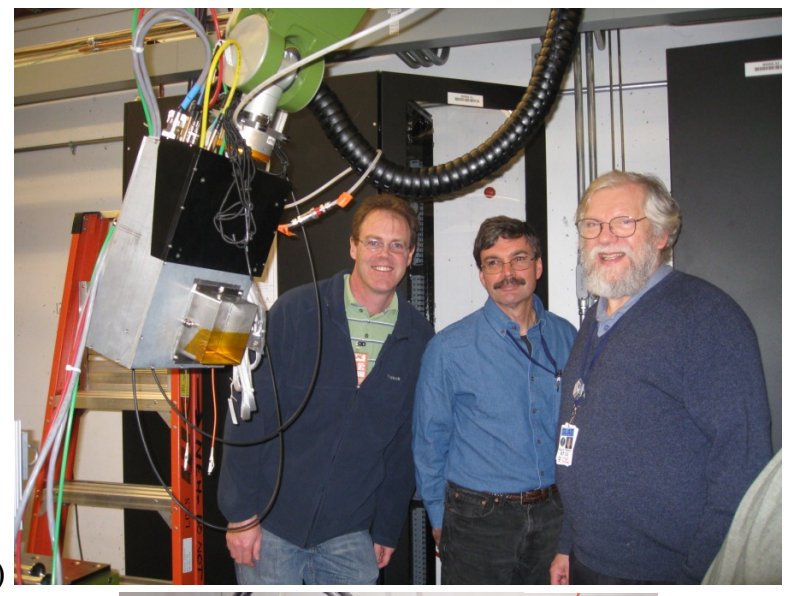

b)

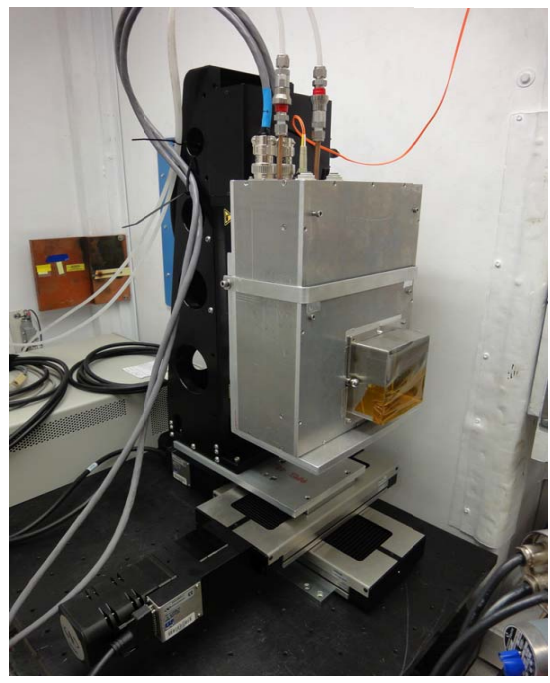

Fig. 1. a) XAMPS system at LCLS XPP; b) XAMPS system at SSRL BL $2-2$.

An x-ray absorption image of a Sequoia sempervirens is shown in Fig. 2. The detector was flood irradiated with the $\mathrm{Cu}$ $\mathrm{K}$-alpha fluorescence emitted by a $\mathrm{Cu}$-foil illuminated with the white beam at SSRL BL2-2. In this image, background subtraction and gain equalization are applied; cosmetic defects are compensated by interpolation. It should be noted that the sensors used to assemble this system don't have a passivation layer and were severely damaged during handling.

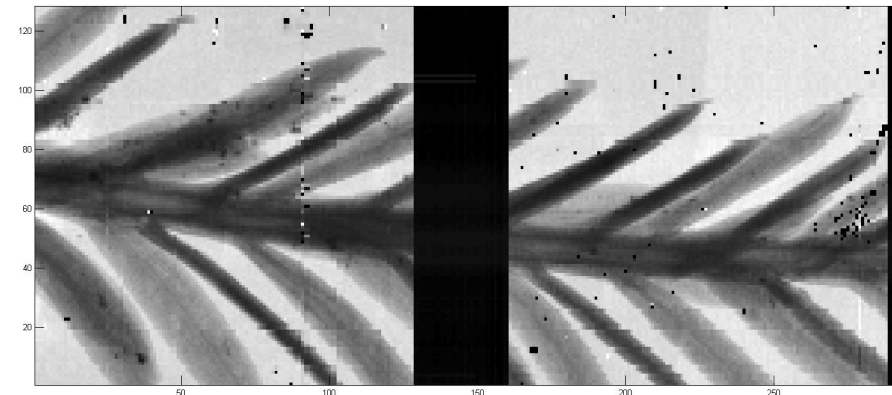

Fig. 2. X-ray absorption image of Sequoia sempervirens: the detector was flood irradiated with the $\mathrm{Cu} \mathrm{K}$-alpha fluorescence emitted by a foil illuminated with white beam. Gain equalization was applied and cosmetic defects compensated by interpolation. 
The features of a California native plant can be appreciated in great detail in Fig. 3. The image was acquired in the same manner: the detector was flood irradiated with the $\mathrm{Cu} \mathrm{K}$-alpha fluorescence emitted by a $\mathrm{Cu}$-foil illuminated with the white beam. This $32 \mathrm{k}$-pixel system was also tested at the LCLS XPP instrument. Fig. 4 shows an image and a correspondent histogram of the LCLS beam.

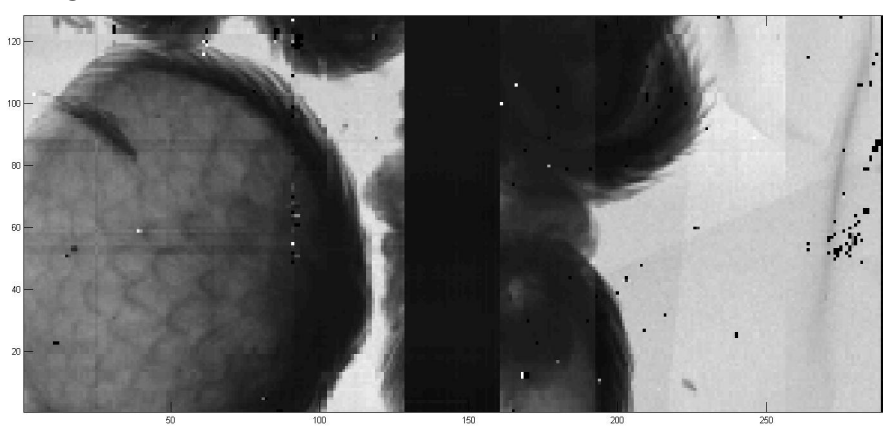

Fig. 3. X-ray absorption image of a California native plant: the detector was flood irradiated with the $\mathrm{Cu}$ K-alpha fluorescence emitted by a foil illuminated with white beam. Gain equalization was applied and cosmetic defects compensated by interpolation.

a)
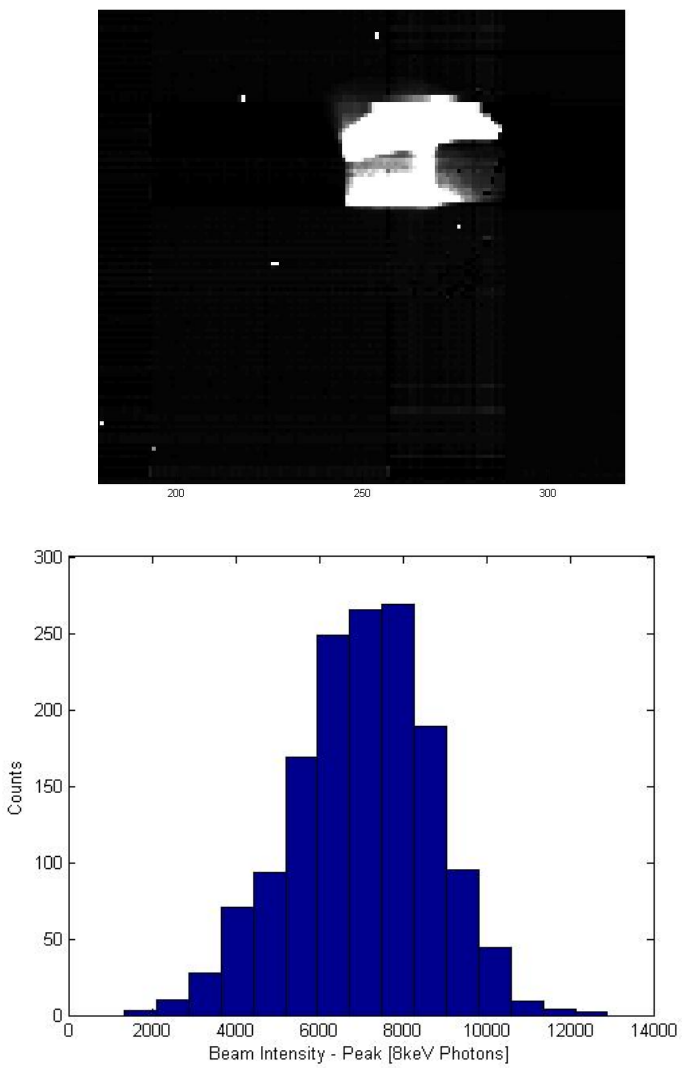

b)

Fig. 4. a) Image of the LCLS beam at XPP: gain equalization was applied and cosmetic defects compensated by interpolation; b) Beam peak intensity average versus number of $8 \mathrm{keV}$ photons.

Only the area of the detector illuminated by the beam is shown in Fig. 4a. Here again, background subtraction and gain equalization are applied and cosmetic defects compensated by interpolation. In Fig. 4b, the histogram of the average beam intensity at its peak position, covering nine pixels, is shown as a function of the number of $8 \mathrm{keV}$ photons. The figure, displaying beam variation, shows the large full-scale signal capability of the system, greater than $10^{4} 8 \mathrm{keV}$ photons/pixel/frame. This is currently the highest value measured with a monolithic silicon pixel detector at LCLS.

\section{HIGH DYNAMIC RANGE BEAM FINDER AT XCS}

A prototype system for monitoring direct beam or single scatter peaks has been developed and tested at the LCLS Xray Correlation Spectroscopy (XCS) instrument. The system is built on the eLine10k test board, a simplified version of the XAMPS system analog board. It mounts one $5 \mathrm{~mm} \times 5 \mathrm{~mm}$ and three $2.1 \mathrm{~mm} \times 1.1 \mathrm{~mm}$ silicon diodes. The setup is completed by a pair of slits, centered on the large diode and mounted on the detector enclosure (Fig. 5).

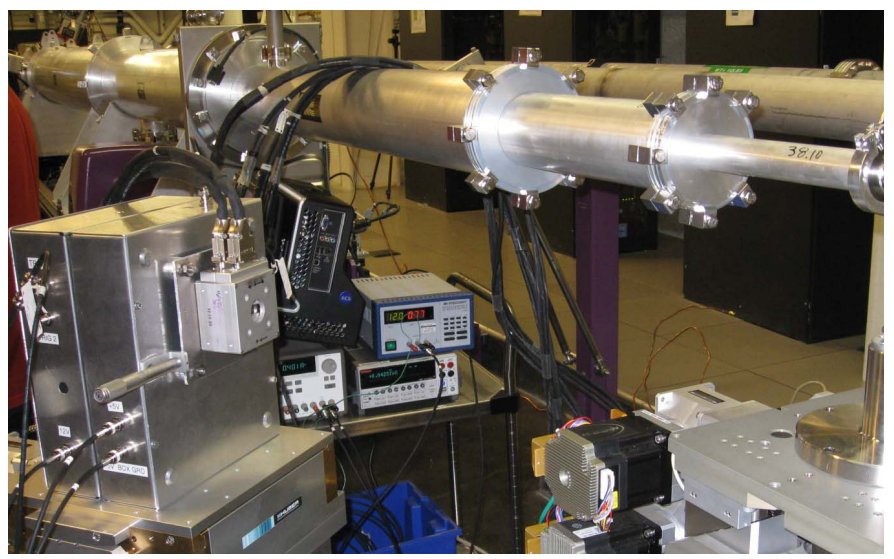

Fig. 5. eLine10k beam finder prototype setup at the XCS instrument.

The slits can be used to raster scan the diode and precisely locate the beam. During this first feasibility study, the detector was illuminated with flood field $\mathrm{Cu}-\mathrm{K}$ photons.

Fig. 6 shows a single photon measurement from the LCLS beam at the XCS instrument. Although the resolution was limited by the sensor leakage current, single photon peaks are clearly distinguishable.

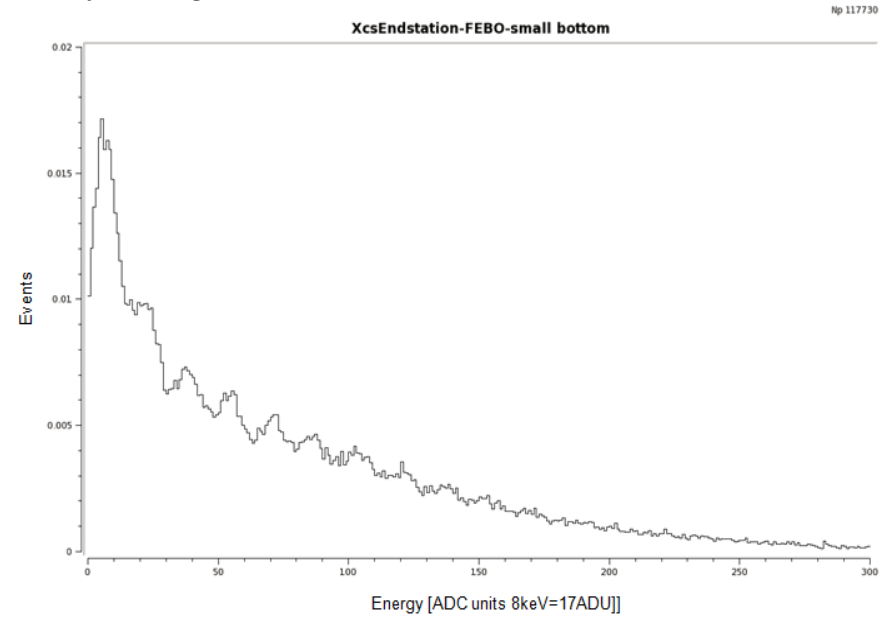

Fig. 6. Single photon measurements: $\mathrm{Cu}$ K-alpha fluorescence measured at the XCS instrument at LCLS. 


\section{SPECTROMETER}

1D and 2D detectors with low noise capabilities are required in a spectrometer configuration in many experiments at LCLS. Detectors for this application should be able to detect very low signal. Therefore, a system developed around the low noise ASIC of the eLine class could provide the required performance. A prototype system consisting of a silicon strip sensor with active edges mounted on the eLine100 test board (a modified version of the XAMPS system analog board) was developed and tested (Fig. 7). The sensor chip measures $3.4 \mathrm{~mm} \times 2.4 \mathrm{~mm}$. It is divided into sixteen strip elements and enclosed by a plasma-diced doped edge [6]. A Peltier-based cooling system was installed on the test board.

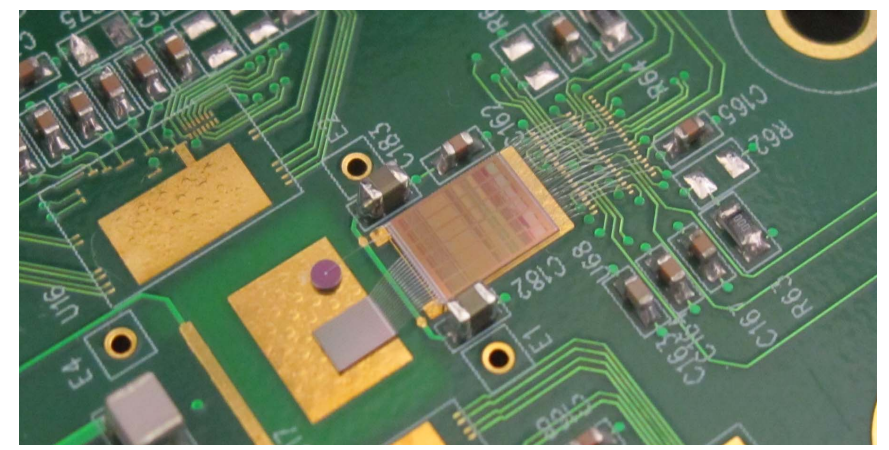

Fig. 7. eLine100 spectrometer: detail of the sensor-ASIC assembly.

A study of the spectroscopic performance of this system was performed and results are reported in Fig. 8. The figure shows ${ }^{55} \mathrm{Fe}$ and ${ }^{109} \mathrm{Cd}$ emission spectra and single $8 \mathrm{keV}$ photons from the fluorescence of a copper target acquired at a sensor temperature of $6^{\circ} \mathrm{C}$ and with an integration time of $32 \mu \mathrm{s}$. The ENC, measured on bonded channels, is as low as $66 \mathrm{e}^{-}$r.m.s., which corresponds to a resolution of $560 \mathrm{eV}$. The spectra are cumulative of the 16 strips and do not implement any split events correction.

\section{CONCLUSIONS}

A new class of ASICs, eLine, for the readout of columnparallel readout sensors has been designed to satisfy the demanding experiments at LCLS. The class is composed of two front-end ASICs and a dedicated controller. eLine10k is the front-end ASIC tailored for high dynamic range applications, optimized for XAMPS detectors and the requirements of the XPP experiment at LCLS. eLine100 is the front-end ASIC tailored for ultra-low noise applications, optimized for XCPS detectors and the requirements of the XCS experiment at LCLS. Prototype systems developed around these two ASICs have been tested at SSRL and LCLS showing the full spectrum of performance and potential applications.

\section{ACKNOWLEDGMENTS}

Portions of this research were carried out at the Stanford Synchrotron Radiation Lightsource and at the Linac Coherent Light Source (LCLS) at the SLAC National Accelerator
Laboratory. SSRL and LCLS are Office of Science User Facilities operated for the U.S. Department of Energy Office of Science by Stanford University.

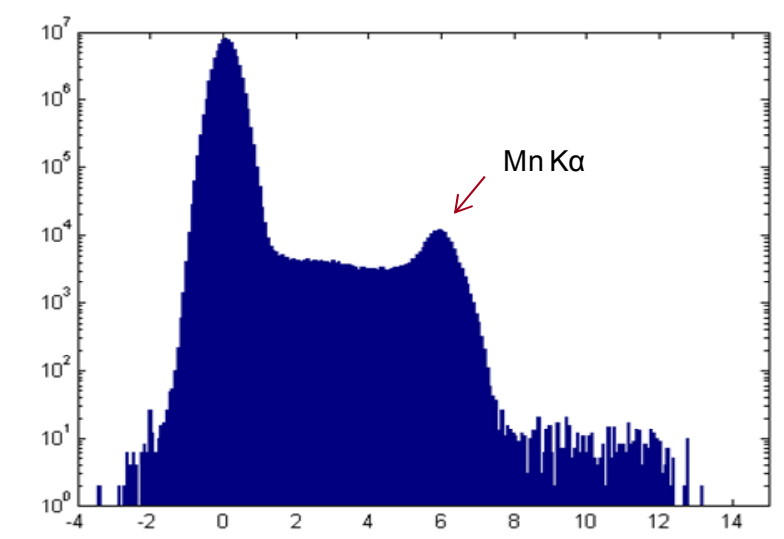

a)

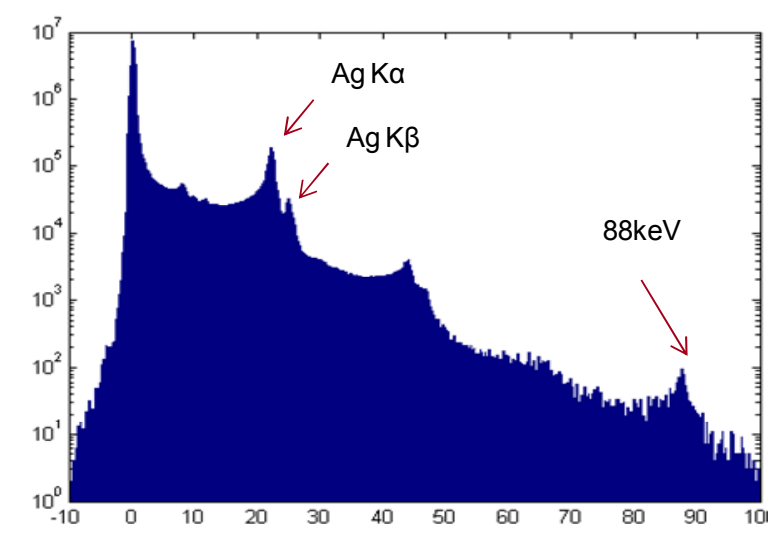

b)

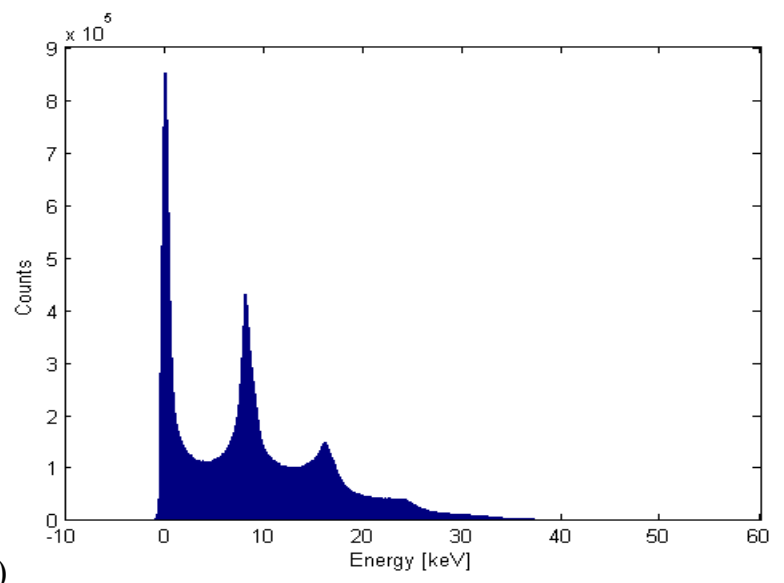

Fig. 8. ${ }^{55} \mathrm{Fe}$ (a) and ${ }^{109} \mathrm{Cd}$ (b) emission spectra acquired at a sensor temperature of $6^{\circ} \mathrm{C}$ and with an integration time of $32 \mu \mathrm{s}$. Mn $\mathrm{K} \alpha$ and $\mathrm{Ag} \mathrm{K \alpha}$, $\mathrm{Ag} \mathrm{K} \beta$ as well as the $88 \mathrm{keV}$ gamma ray can clearly be observed in (a) and (b) respectively. (c) Single $8 \mathrm{keV}$ photons from the fluorescence of a copper target.

\section{REFERENCES}

[1] A. Dragone, P. Caragiulo, G. A. Carini, R. Herbst, J.F. Pratte, P. O'Connor, P. Rehak, D. P. Siddons and G. Haller, "eLine10k: An High Dynamic Range Front End ASIC for LCLS Detectors", to appear on NSS-MIC 2012 conference records. 
[2] A. Dragone, J.F. Pratte, P. Rehak, G. A. Carini, R. Herbst, P. O'Connor, D. P. Siddons, "XAMPS Detectors Readout ASIC for LCLS", Nuclear Science Symposium Conference Record (NSS/MIC), 2970 (2008).

[3] A. Dragone, P. Caragiulo, D. Freytag, R. Herbst, C. Kenney, J. Segal, G. Haller, "eLine100: A Front End ASIC for LCLS Detectors in Low Noise Applications", to appear on NSS-MIC 2012 conference records.

[4] W. Chen, G. De Geronimo, Z. Li, P. O'Connor, V. Radeka, P. Rehak, G. C. Smith, B. Yu, IEEE Trans. Nucl. Sci. 49 (3) (2002) 1006.

[5] G. A. Carini, W. Chen, A. Dragone, J. Fried, J. Jakoncic, A. Kuczweski, Z. Li, J. Mead, R. Mitcha, J.-F. Pratte, P. Rehak, D. P. Siddons, "Tests of small X-ray Active Matrix Pixel Sensor prototypes at the National Synchrotron Light Source", 2009 JINST 4 P03014.

[6] G. A. Carini, A. Dragone, W. Chen, J.Fried, A. Kuczewski, Z. Li, J. Mead, P. O'Connor, J-F Pratte, P. Rehak, K. Wolniewicz and D. P. Siddons, "The XAMPS detector for the X-ray Pump-Probe instrument at LCLS", IEEE Nuclear Science Symposium Conference Records. 21512153 (2009).

[7] C. J. Kenney, J. D. Segal, E. Westbrook, S. Parker, J. Hasi, C. Da Via, S. Watts, J. Morse, "Active-edge planar radiation sensors", Nucl. Instr, Meth. A, 565 (1) (2006) 272. 